\title{
Designing a Partly Self-Powered Keyboard Based on Triboelectric Effect
}

\author{
Hoang Si Hong*, Nguyen Dinh Minh, Nguyen Duc Thuan, \\ Nghiem Thi Hai, Dinh Xuan Trung Duc, Le Viet Toan \\ School of Electrical Engineering, Hanoi University of Science and Technology, Hanoi, Vietnam \\ *Email: hong.hoangsy@hust.edu.vn
}

\begin{abstract}
The purpose of this paper is to design a keyboard using the triboelectric effect (Tribo Electric Nano Generator - TENG) to collect a part of the energy from keystrokes to reduce the power consumption of the keyboard. Using elastic material as the cover on the keyboard to maximize the capture of energy from typing. The keyboard layers are made from common materials such as Al (Aluminum) and PTFE (Polytetrafluoroethylene). The built-in 16-button keyboard ensures the same typing speed as a typical keyboard. Based on selected triboelectric material, the output voltage of keyboard was simulated and processed by using a signal detection circuit. The results show that the average voltage generated by each key with electrical friction effect is about $4 \mathrm{~V}$, the power consumption for the detection circuit is about $0.32 \mathrm{~W}$. In addition, the keystroke signals were sent and displayed correctly on the designed software on the computer.
\end{abstract}

Keywords: Triboelectric, keyboard, self-powered.

\section{Introduction}

Recently, the research on self-powered devices has been receiving much focus by researchers in the field. As such, harvesting energy based on the Triboelectric effect becomes a potential research topic. The fundamental principle of TENGs is based on contact electrification and electrostatic induction. When two surfaces of different materials come into contact, they form a chemical connection that makes electrons travel between two surfaces in order to reach the balance in the electrochemical state. This phenomenon creates an electric imbalance between materials. Connecting two electrodes to a load, a current will be formed. Besides, there was much research in this field that tried to improve the efficiency of the energy harvesting procedure [1]. Therefore, the Triboelectric effect is applicable for containing energy generated from human body movements, then uses this energy to partly provide power for charging mobile devices, smartphones [1, 2].

Among Triboelectric-based applications, the self-powered technique is the most concerning field. Shengming $\mathrm{Li}$ and Wenbo Peng [3] researched harvesting energy by typing a computer's keyboard. Xindia Wang [4] focuses on the matrix of the triboelectric node (triboelectric generator). These two papers have eyes on the productivity of energy captivating by typing in different conditions and storing them.

However, these results $[3,4]$ did not propose any complete applications. Hence, this paper proposes a complete keyboard design which can partly supply itself by typing activity based on the Triboelectric effect. To design this keyboard, Section 2 shows the fundamental theories of the triboelectric effect. Using the derived equation, real-time node output characteristics are mathematically modeled and simulated. The design of the signal processing circuit and the measured results of the node and the whole system will be shown in Section 3.

\section{Modeling and Designing}

\subsection{Theory}

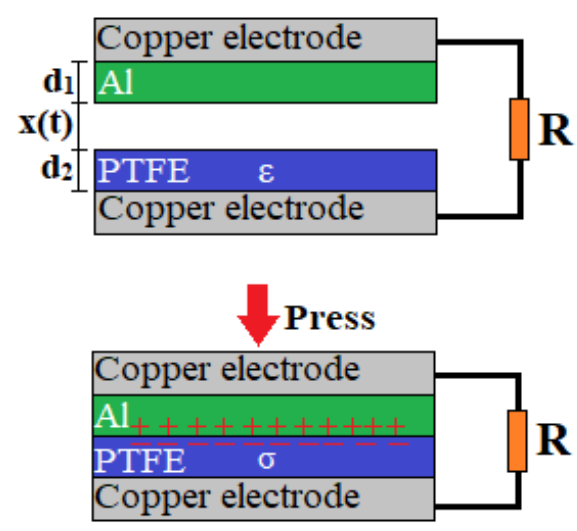

Fig. 1. Theory of triboelectric generator.

A simple triboelectric generator consists of two layers of triboelectric material and electrodes attached on the outer side of each triboelectric layer, as shown in Fig. 1. When two layers come into contact, they establish a chemical bond between the surfaces of the 
two layers. Electrons travel from one layer to the other to balance chemical potential. Thus, they get charged and an electric potential is created between two surfaces. Copper electrodes are attached to the outer side of each triboelectric layer. These electrodes connect with the signal processing circuit.

To be more specific, the distance between the two triboelectric layers $(x)$ can be varied whether the node is pressed. While two triboelectric layers are forced to get in contact with each other, the inner surfaces of them will have opposite static charges with an equal density of $s$, resulting in contact electrification. After separating from each other, with increased $x$, a potential difference $(V)$ between the two electrodes will be induced. $Q$ is the number of transmitted charges between the two electrodes, as driven by the induced potential, which also represents the instantaneous amount of charges on each electrode. Thus, the $V-Q-x$ relationship is the most important theoretical equation for representing the real-time power generation of a triboelectric node. In electrification theory, $V-Q-x$ relationship energy gaining from the triboelectric node is derived from the $V-Q-x$ relationship: [5]

$$
V=-\frac{1}{C_{T E N G}} Q+V_{O C}
$$

where $V_{O C}$ is the potential between two triboelectric layers, $C$ is the capacitance of two electrodes [6].

In (1), on the right side, there are two voltage terms: one is the voltage originated from two electrode plates, and the other is the open-circuit voltage generated from the separation of two triboelectric layers. $V_{O C}$ và $C_{T E N G}$ is the function of the air gap $(x)$ between two triboelectric layers. Besides, the characteristic of the output in (1) is dependent on the velocity and acceleration of the separation. To simulate the triboelectric generator with resistance load in MATLAB, some hypotheses were proposed [5]. The separation of two triboelectric layers is the constant motion, hence:

$$
x=x_{0}+v t .
$$

The capacitance of the generator:

$$
C_{\text {TENG }}=\frac{S \varepsilon_{0}}{d_{0}+x(t)}
$$

where $\varepsilon_{0}=8,85 \cdot 10^{-12}$ is the absolute dielectric constant, $d_{0}=\frac{d}{\varepsilon}$ with $d, \varepsilon$ is the thickness and dielectric constant of dielectric layers, respectively. Open-circuit voltage is:

$$
V_{O C}=\frac{\sigma x(t)}{\varepsilon_{0}}
$$

where $\sigma$ is the surface charge density of dielectric layers.
Equation of triboelectric sensors and resistance load [5] is:

$$
R \frac{d Q}{d t}=V=-\frac{1}{C_{T E N G}} Q+V_{O C}=-\frac{d_{0}+x(t)}{S \varepsilon_{0}} Q+\frac{\sigma x(t)}{\varepsilon_{0}}
$$

Equation (5) is a first-order ordinary differential equation and can be solved by specifying the boundary condition. Consider the case that at $t=0$, the two triboelectric layers are close for a sufficient time and then the top and bottom plate starts to separate from each other. With the boundary condition of $Q(0)=0$, solving (5), we have:

$$
\begin{aligned}
Q(t)= & \frac{1}{R} \exp \left[-\frac{1}{R} \int_{0}^{t} \frac{1}{C(x(t))} d t\right] \\
& \times \int_{0}^{t} V_{O C}(x(t)) \exp \left[\frac{1}{R} \int_{0}^{t} \frac{1}{C(x(t))} d t\right] d t
\end{aligned}
$$

Therefore, the current and voltage output can be caculated as:

$$
\begin{aligned}
V(t)= & V_{O C}-\frac{1}{R C} \exp \left[-\frac{1}{R} \int_{0}^{t} \frac{1}{C(x(t))} d t\right] \\
& \times \int_{0}^{t} V_{O C}(x(t)) \exp \left[\frac{1}{R} \int_{0}^{t} \frac{1}{C(x(t))} d t\right] d t \\
I(t)= & \frac{V_{O C}}{R}-\frac{1}{R^{2} C} \exp \left[-\frac{1}{R} \int_{0}^{t} \frac{1}{C(x(t))} d t\right] \\
& \times \int_{0}^{t} V_{O C}(x(t)) \exp \left[\frac{1}{R} \int_{0}^{t} \frac{1}{C(x(t))} d t\right] d t
\end{aligned}
$$

The above equations indicate that the transferred charge, voltage, and current are directly proportional to the tribo-charge surface density $(\sigma)$. Thus, $\sigma$ will only affect the magnitude of these parameters but not their variation trend and shape with both time and $R$. $\sigma$ is mainly affected by the characteristics of triboelectric layers and roughness of the contact surfaces. Therefore, through choosing materials, $\sigma$ can be increased and then the performance of the node can be enhanced. In this experiment, the material PTFE is chosen and $\sigma$ is estimated at about $20 \mu \mathrm{Cm}^{-2}$.

\subsection{Simulation and Selecting Structure Parameter of the Nanogenerator}

In order to design a keyboard based on the triboelectric effect, it is necessary to calculate and optimize: the node size, the distance between two triboelectric layers, the input impedance of the signal processing circuit. This can be done by simulating. the impact of these parameters on the output of the node.

In reality, the connection between triboelectric layers and dielectric layers is not ideal, thus voltage loss will be formed. From the result in (7), $Z_{t t}$ is the internal impedance of each key, assume that operating velocity is constant, the output voltage is: 


$$
V(t)=\frac{R}{R+Z_{t t}}\left[\frac{\sigma v t}{\varepsilon_{0}}-\frac{d_{0}+v t}{\left(R+R_{t t}\right) S \varepsilon_{0}} \exp \left[-\frac{1}{R+Z_{t t}} \int_{0}^{t} \frac{d_{0}+v t}{S \varepsilon_{0}} d t\right] \int_{0}^{t} \frac{\sigma v t}{\varepsilon_{0}} \exp \left[\frac{1}{R+Z_{t t}} \int_{0}^{t} \frac{d_{0}+v t}{S \varepsilon_{0}} d t\right] d t\right]
$$

Table 1. Reference parameter in simulation

Parameter

Symbols

Value

\begin{tabular}{lcc}
\hline AI thickness & $d 1$ & $100 \mu \mathrm{m}$ \\
PTFE thickness & $d 2$ & $100 \mu \mathrm{m}$ \\
Dielectric constant of PTFE layer & $\varepsilon$ & $2.05[3]$ \\
Surface charge density & $\sigma$ & $20 \mu \mathrm{Cm}^{-2}$ \\
Maximum distance between two tribo layers & $x_{\max }$ & $1 \mathrm{~mm}$ \\
Resistance & $R$ & $100 \mathrm{M} \Omega$ \\
generator size & $a$ & $1.5 \mathrm{~cm} \mathrm{x} 1.5 \mathrm{~cm}$ \\
Velocity & $v$ & $0.1 \mathrm{~m} / \mathrm{s}$ \\
\hline
\end{tabular}

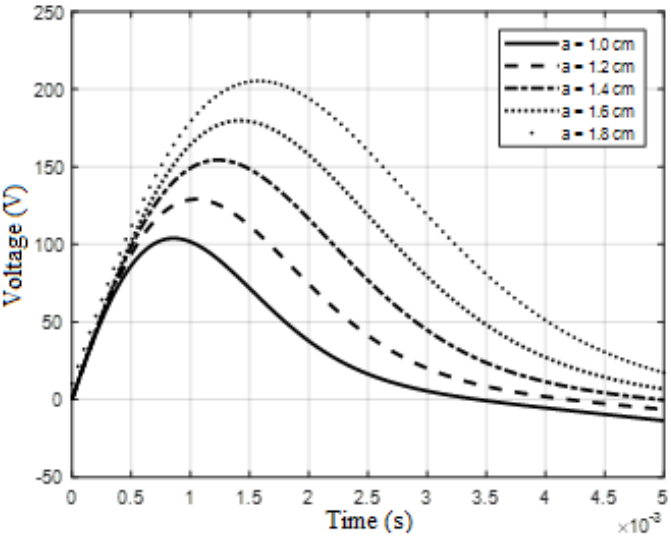

Fig. 2. Simulating the relationship between node size and ouput voltage.

In all the simulations, this paper idealizes $R_{t t}=0$ and other parameters are taken from Table 1 . Observing the results obtained from Fig. 2, node size has a great impact on the output voltage. Specifically, the greater the node size, the greater voltage generated, thus easier for harvesting and processing. However, due to the limited size of the key, from Fig. 2, the node was chosen with a size of $1.5 \mathrm{~cm} \times 1.5 \mathrm{~cm}$ to ensure compact level.

Besides node size, the air-gap or distance between two triboelectric layers, $x_{\max }$, is an important factor. As shown in Fig. 3, the greater distance $x_{\max }$ is, the smaller capacitance becomes, which makes the generated voltage becomes higher. Therefore, the optimal distance for the node is a minimum of $1 \mathrm{~mm}$, and to ensure the node size is compact, this paper chose $x_{\max }=1 \mathrm{~mm}$ for reference and design.

The output voltage will not decrease if the load increases (shown in Fig. 4). However, an extremely high resistance load allows a really low current to flow

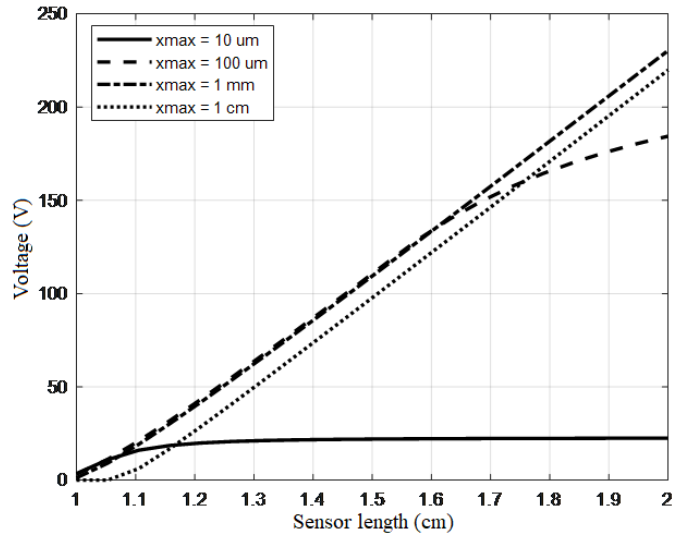

Fig. 3. Simulating the relationship between distance and output voltage.

through or approximately $0 \mathrm{~A}$, thus instantaneous power cannot reach maximum.

Fig. 5 indicates that as for typing speed, with high velocity, the output voltage reaches the maximum state fast, then gets to the negative value. The reason behind that is while operating with high velocity, $x$ quickly reaches its maximum; $V_{O C}$ does not change, but the differential component of $C_{\text {TENG }}$ pulls the output voltage to a negative value.

\subsection{Fabrication of triboelectric node and valuation}

Some of the requirements that triboelectric effect based self-powered node needs to meet are high sensitivity, flexibility, and easy to produce [4]. Therefore, this paper chooses to use PTFE and AI as main materials. According to the results from Fig. 2, Fig. 3, Fig. 4, and Fig. 5, some parameters of the node are taken from Table 1 for node manufacturing. Node size (a): $1.5 \mathrm{~cm} \times 1.5 \mathrm{~cm}$; distance between two triboelectric layers $\left(x_{\max }\right): 1 \mathrm{~mm}$, thickness of PTFE layer (d2): $100 \mu \mathrm{m}$. 


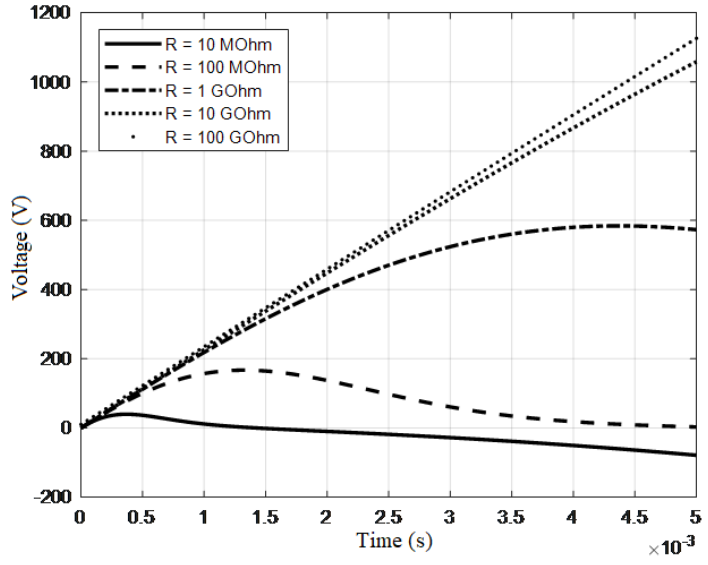

Fig. 4. Simulating the relationship between velocity and output voltage.

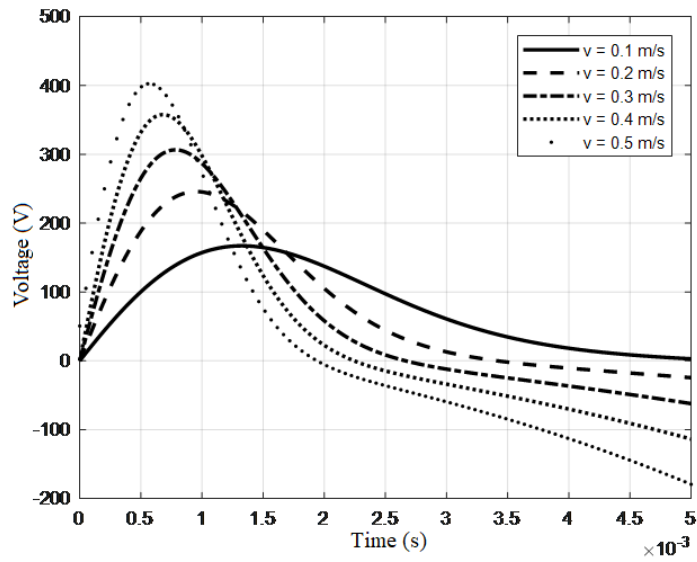

Fig. 6. Simulating the relationship between velocity and output voltage.

Fig. 6 illustrates the structure of the node and its layer. The node has four layers including: two copper electrode layers placing on the top and bottom side; the Aluminum layer acting as a positive triboelectric layer; and PTFE layer as a negative triboelectric layer. Between two of these layers is the air gap and the completed node is placed on a PCB base. Layers are bonded by surface connection. Thus, mechanical solidarity is limited. Besides, a silicon layer covers the node in order to ensure stability, longevity, and water resistance as well as decreasing the effect of the outside environment and human skin.

Fig. 7 shows the actual view of the sensor while two layers are left unfold. the film above was folded double to form a square shape to create a complete node where a layer of negative materials was overlapped each other.

In reality, one out of two triboelectric materials is not conducting electricity (PTFE). So, this layer has a high internal impedance. Thus, the real output voltage gained from the node is only similar to the form of simulating output voltage. With $Z_{t t}=2 \mathrm{G} \Omega$, close to the actual condition, Fig. 8 illustrates the actual output

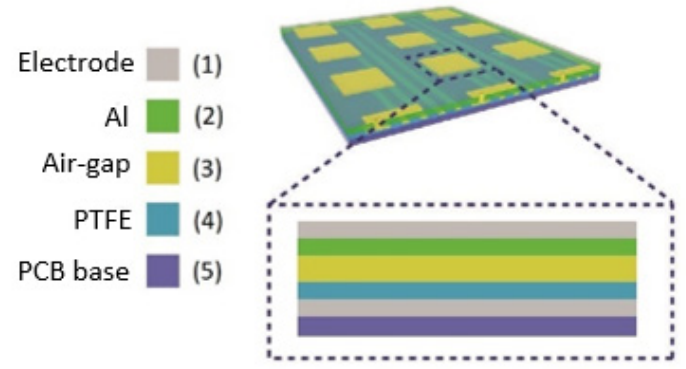

Fig. 5. Structure of the triboelectric node

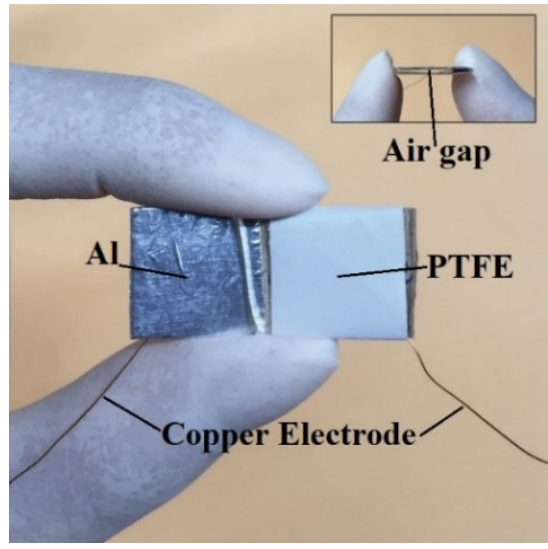

Fig. 7. Actual triboelectric node.

voltage form is similar to the simulation. Since $Z_{t t}=2 \mathrm{G} \Omega$ is only the average assumption, $V \max$ has not got the calculated value. This creates trouble and challenge for handling signal process. The actual output of the node and system was measured by a TDS1001c-edu oscilloscope and will be discussed in more detail in section 3 .

\section{Results and discussion}

\subsection{Signal analysis and signal detection circuit}

Since the original signal obtained from the sensor has sine waveform, low energy, and easily being affected by nois, directly extracting features from the sensor results in low accuracy. Thus, a standardized circuit to detect signals is necessary. The signal detection circuit converts the original signal to a pulse waveform with a similar amplitude.

Also, the voltage and current waveform generated from the triboelectric-based sensor are sine waves with (Positive and negative period). A full-wave rectifier was used to optimize energy gained from the triboelectric node, then capacitors are used to store this energy (Fig. 9) [8]. Regarding this method, it is 
important to calculate a proper rectifier, and capacitors need to be adjusted depending on the actual signal.

Fig. 10a illustrates the original signal from the triboelectric node by typing slowly (10-20 s per press). Fig. $10 \mathrm{~b}$ is the detail of one chunk of signal from Fig. 10a. Keys are pressed slowly (10-20 s per press) to clearly see the useful signal and noise signal.

The original signal after going through the rectifier has a pulse waveform. To obtain the peak value of these pulses, an envelope detector was used. A simple circuit is shown in Fig. 11. In addition to triboelectric noise, human hands can trigger signals to high value. Thus, the load of the peak detector is a potentiometer in order to get $3.3 \mathrm{~V}$ output voltage. The output signal of the peak detector is the input of Trigger Schmitt as shown in Fig. 11. The output of Inverting Trigger Schmitt is connected to a noninverting pin of the comparator. This creates a delay comparator circuit with a threshold of $0 \mathrm{~V}$, and the pulse width is determined by the ratio of $\mathrm{R} 11, \mathrm{R} 12$, and R13.

The input value of Trigger Schmitt is calculated and based on the threshold of the triboelectric signal after going through the unity-gain bandwidth amplifier.

Fig. 11 describes the scheme of the signal detector circuit of a triboelectric node. The important factor to determine the threshold is the initial noise voltage of the triboelectric signal when there is no external force applied. According to the actual result, the noise signal is around $1 \mathrm{~V}$, thus the $3.3 \mathrm{~V}$ threshold is proper to avoid comparing with noise. From experiment results, the value of $\mathrm{R} 11, \mathrm{R} 12$, and $\mathrm{R} 13$ is $2.5 \mathrm{~K}, 10 \mathrm{~K}$, and $12 \mathrm{~K}$ respectively.

Fig. 12 illustrates the output voltage of signal detector circuit when repeatedly presses one key. According to Fig. 12, the time of pressing and releasing one key is around $0.2 \mathrm{~s}$. That means this system can receive a maximum of 300 signals per minute, which equals the standard tying speed.

The output above then goes through a microcontroller to convert the signal to the standard output of a keyboard and be able to connect to a laptop via a USB interface, as shown in Fig. 13.

Fig. 14 illustrates the actual signal processing circuit for a keyboard. Circuit uses conventional IC, therefore, compared to the keyboard using specific IC: the experiment's actual circuit is bigger. The output signal is connected to the PC via a USB port as the common keyboard. In this work, the keyboard signal sent to the PC is read by Hercules software as shown in Fig. 15.

Fig. 16 illustrates the structure of a conventional capacitance keyboard. To operate conventional keyboard, energy is supplied to the capacitor, oscillator, phase-locked loop, and comparator [9]. On the other hand, in a Triboelectric keyboard, a fraction of energy is provided by typing action. Hence, only the comparator needs to be supplied. As a result, the Triboelectric keyboard consumes less energy than a common keyboard does.

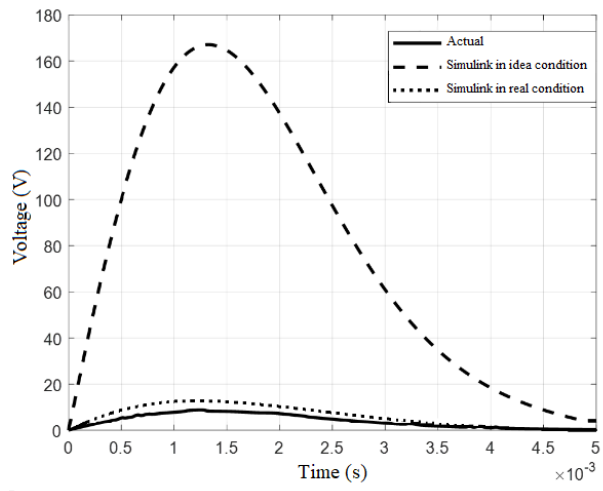

Fig. 8. Simulating output and actual output

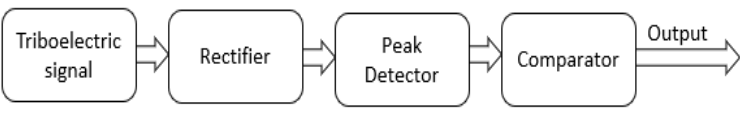

Fig. 9. Signal detector circuit diagram
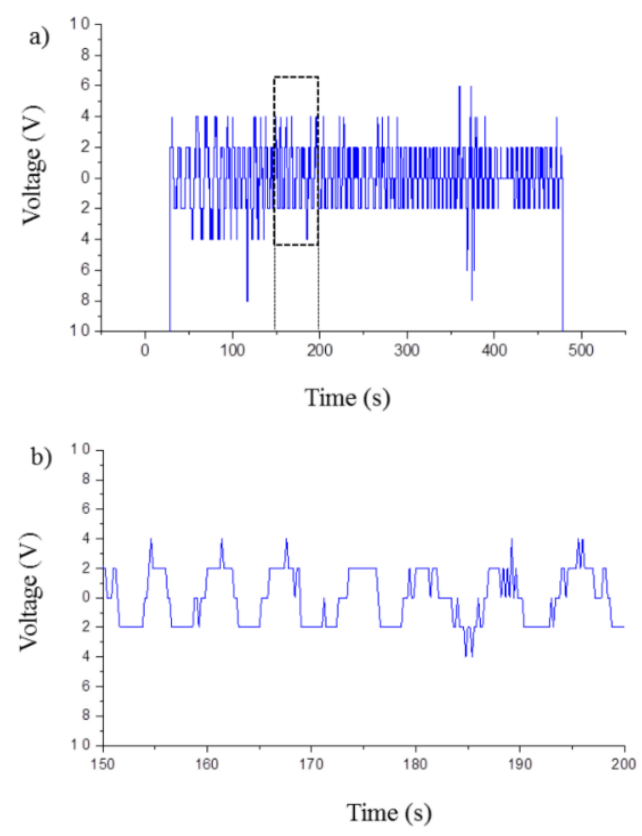

Fig. 10. The output voltage of sensor.

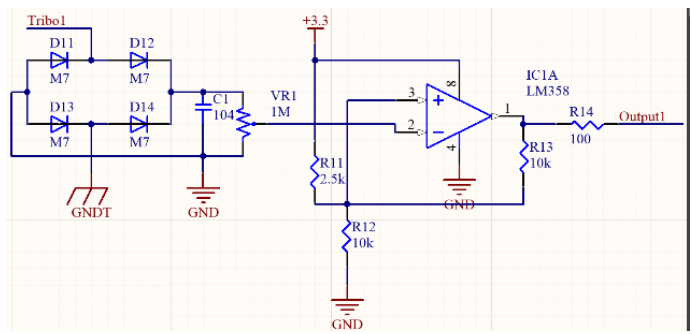

Fig. 11. Signal detector circuit of a triboelectric node. 


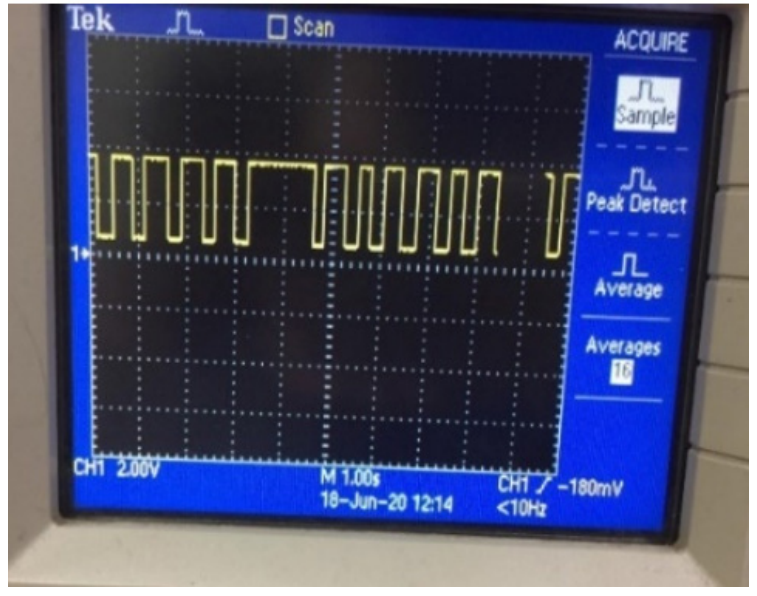

Fig. 12. The output signal of signal detector circuit

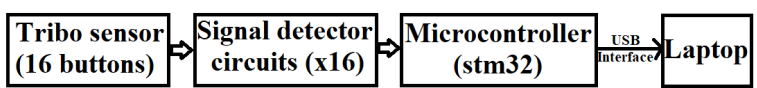

Fig. 13. Keyboard diagram.

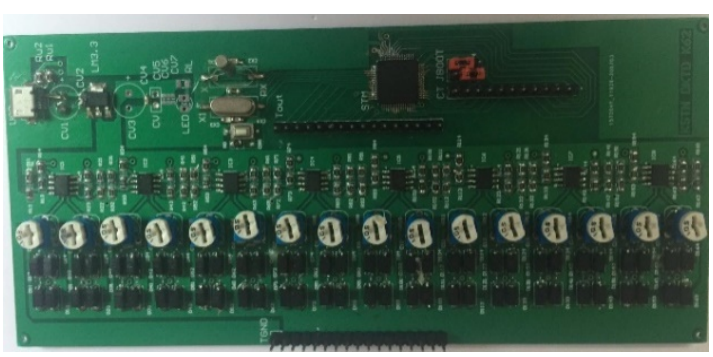

Fig. 14. Actual circuit.

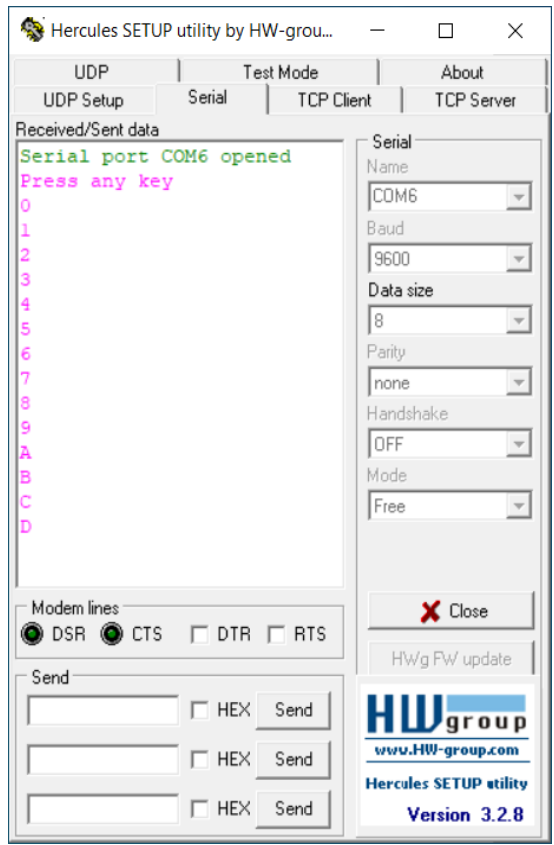

Fig. 15. Keyboard signal presents on PC.

\section{Keyboards}
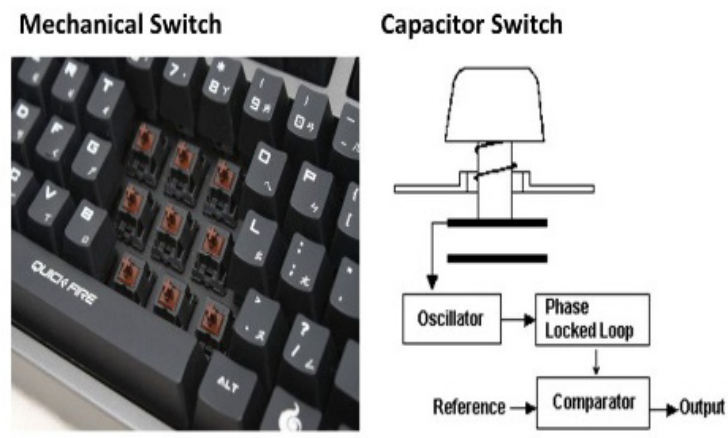

Fig. 16. Structure of conventional keyboard.

\subsection{Power analysis}

As both this experiment's keyboard and conventional keyboard connect to a laptop via USB interface, a USB tester can measure the current, voltage and power of keyboard while keeping data transfer between keyboard and laptop active. Fig. 17 shows the cabling diagram to measure the power consumption of the keyboard in normal working conditions.

A USB tester FNB38 consists of USB-A, MicroUSB, and Type-C connections. It uses ADC, PD 16 bit. Power range $0-120 \mathrm{~W}$, power resolution $0.1 \mathrm{~mW}$ and precision $\pm\left(0.5 \%{ }_{0}+2\right)$ [4]. The FNB38 Power Meter's internal logical structure is presented in Fig. 18. There are three main components voltage and measurement chip, OLED display, and microcontroller unit which communicate with each other using the $\mathrm{I} 2 \mathrm{C}$ communications protocol. The voltage/current measurement chip and the OLED display represent the slaves, the microcontroller represents the master controlling slaves through GPIO gates.

Fig. 19a shows the output power of a conventional keyboard, the power is around $0.54 \mathrm{~W}$. Fig. 19b illustrates the output power of a keyboard operating based on triboelectric nodes, the power is around $0.32 \mathrm{~W}$. The designed keyboard has 16 buttons equivalent to 16 signal nets connecting to the main comparator and processor whereas the conventional keyboard also has 16-19 signal nets depending on manufacturer. Therefore, the comparison of the power consumption of the two keyboards does not lose its generality. In this paper, the handmade PCB cannot optimize output power, and using microcontrollers consume more energy than the logic circuit. However, the power consumption is only $2 / 3$ compared to that of a conventional keyboard. This shows a promising development of the keyboard based on triboelectric effect. 


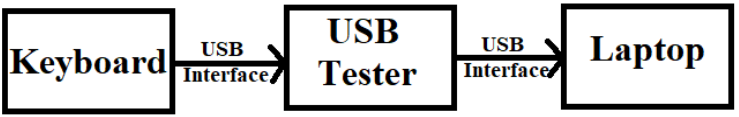

Fig. 17. Cabling diagram

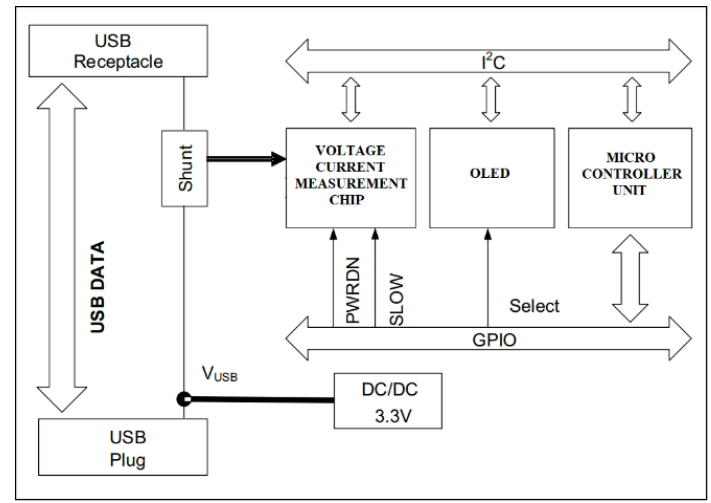

Fig. 18. Block diagram of Current and Voltage Meter USB Tester.

\section{Conclusion}

This research successfully designed a keyboard based on triboelectric effect and survey its characteristics. The triboelectric node is made out of sustainable, flexible materials (PTFE and Aluminum) with proper sensitivity. The power consumption of our keyboard is low at $0.3227 \mathrm{~W}$. Thus, this research is an early step toward producing a self-powered keyboard based on the triboelectric effect.

However, in this work, the sensitivity of the keyboard is just enough, enduration is not good enough for commerce. In order to improve sensitivity and enduration, instead of PTFE, the keyboard can be made out of advanced materials such as polyurethane foam, polydimethylsiloxane. In further research, the experiment about the correlation between typing force and performance of the developed keyboard should be conducted. More test scenarios are needed to assess the keyboard's reliability and energy savings.

\section{Acknowledgment}

This research is funded by Vietnam National Foundation for Science and Technology Development (NAFOSTED) under grant number 103.02-2018.33.

\section{References}

[1]. S. Niua and Y. S. Zhou, Simulation method for optimizing the performance of an integrated

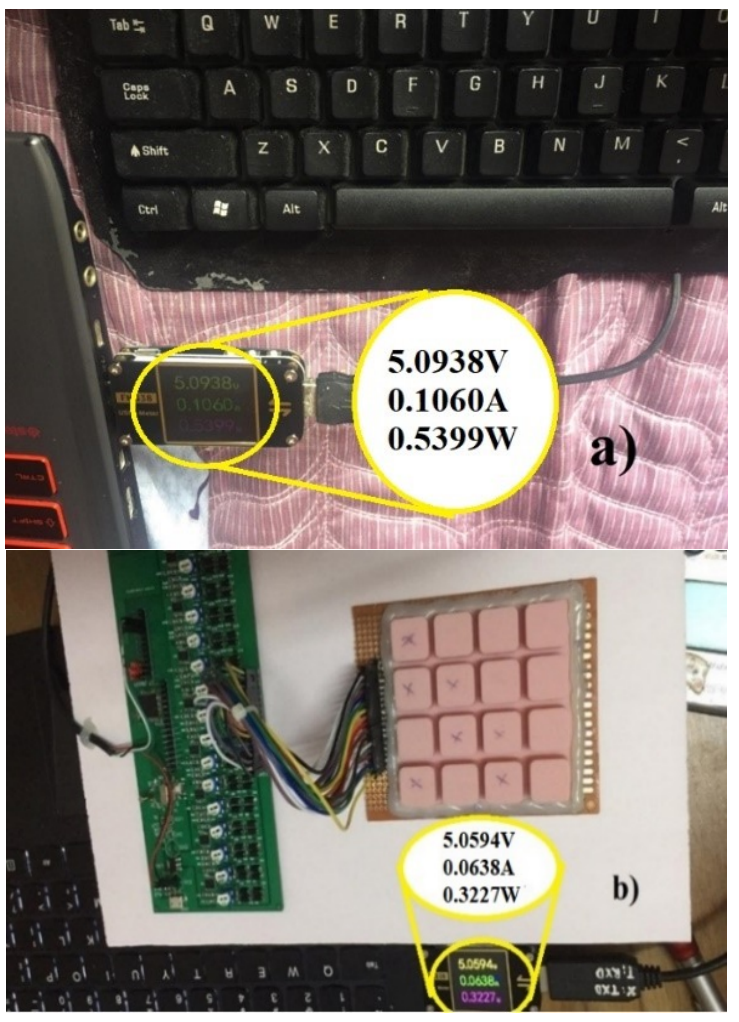

Fig. 19. Keyboard output power.

triboelectric nanogenerator energy harvesting system, Nano Energy, vol. 8, pp. 150-156, Jun. 2014 https://doi.org/10.1016/j.nanoen.2014.05.018

[2]. S. Niu and Z. L. Wang, Theoretical systems of triboelectric nanogenerators, Nano Energy, vol. 14, pp. 161-192, May. 2015,

https://doi.org/10.1016/j.nanoen.2014.11.034

[3]. G. Singh, Measurement of dielectric constant and loss factor of the dielectric material at microwave frequencies, Progress In Electromagnetics Research, vol. 69, pp. 47-54, 2007 https://doi.org/10.2528/PIER06111204

[4]. X. Li and C. Jiang, Fully stretchable triboelectric nanogenerator for energy harvesting and self powered sensing, Nano Energy, vol. 61, pp. 78-85, Jul. 2019 https://doi.org/10.1016/j.nanoen.2019.04.025

[5]. F. Xi and Y. Pang, Universal power management strategy for triboelectric nanogenerator, Nano Energy, vol. 37, pp. 168-176, Jul. 2017

https://doi.org/10.1016/j.nanoen.2017.05.027

[6]. S. Li and W. Peng, All-elastomer-based triboelectric nanogenerator as a keyboard cover to harvest typing energy, ACS Nano, vol. 10, no. 8, pp. 1973-1981, Aug. 2016,

https://doi.org/10.1021/acsnano.6b03926

[7]. X. Wang and H. Zhang, Self-powered high-resolution and pressure-sensitive triboelectric sensor matrix for real-time tactile mapping, Advanced Materials, vol. 28 , no. 15 , pp. 2896-2903, Feb. 2016, https://doi.org/10.1002/adma.201503407 
JST: Smart Systems and Devices

Volume 31, Issue 2, September 2021, 035-042

[8]. S. Niu and X. Wang, A universal self-charging system driven by random biomechanical energy for sustainable operation of mobile electronics, Nature Communications, vol. 6, no. 1, Dec. 2015,

https://doi.org/10.1038/ncomms9975
[9]. Fnirsi, FNB38 manual Version 1.2, July. 29, 2020. [Online]. Available: http://fnirsi.cn/support. 